\title{
Emergência de plântulas de Anacardium humile A. St.-Hil. (Anacardiaceae) avaliada por meio de amostras pequenas
}

\author{
MARISTELA P. CARVALHO ${ }^{1}$, DENISE G. SANTANA ${ }^{1,3}$ e MARLI A. RANAL ${ }^{2}$
}

(recebido: 14 de outubro de 2004; aceito: 6 de outubro de 2005)

\begin{abstract}
Anacardium humile A. St.-Hil. (Anacardiaceae) seedling emergence evaluated by means of small samples). Anacardium humile has low capacity for fruit production, limiting the application of experimental techniques, broadly used in the analysis of seed germination. Thus, the purpose of this paper was to introduce the statistical theory of small samples to the study of the seedling emergence of this species. Cashew nuts of different individuals were randomly collected, being submitted to scarification by abrasion of the pericarp near the pedicel; scarification in the same manner followed by washing in water for 24 hours, and intact cashew nuts as control treatment. The cashew nuts (50 per treatment) were sowed at $1 \mathrm{~cm}$ depth, in vermiculite moistened with distilled water at field capacity. The experiment was carried out under continuous fluorescent white lamps $\left(21.8 \mu \mathrm{mol} \mathrm{m} \mathrm{m}^{-2} \mathrm{~s}^{-1}\right)$, at $25^{\circ} \mathrm{C}$. The emergence (\%) presented approximated normal distribution for all treatments and they did not differ significantly by Student $t$ test (emergence between $70 \%$ and $76 \%$ ). Seedlings emerged from scarified cashew nuts washed in water presented shorter mean emergence time (16.3 days) and consequently higher mean emergence rate $\left(0.0613\right.$ day $\left.^{-1}\right)$ in relation to the other treatments. The uncertainty values far from zero ( $I \geq 3.28$ bits) and values of $Z$ near zero $(Z \leq 0.095)$ indicated low seedling emergence synchrony for this species. The results obtained showed that by using reduced number of cashew nuts it was possible to infer about seedling emergence process without violation of the statistical principles and assumptions.
\end{abstract}

Key words - cashew nut, emergence measurements, normal distribution, Student t test

RESUMO - (Emergência de plântulas de Anacardium humile A. St.-Hil. (Anacardiaceae) avaliada por meio de amostras pequenas). Anacardium humile possui baixa capacidade de produção de frutos, o que limita a aplicação de técnicas experimentais, amplamente utilizadas na análise da germinação. Assim, o objetivo do trabalho foi introduzir a teoria estatística de amostras pequenas ao estudo da emergência de plântulas da espécie. Núculas foram aleatoriamente coletadas de diferentes indivíduos, sendo submetidas à escarificação por abrasão do pericarpo na região junto ao pedicelo; escarificação na mesma região, seguida de lavagem em água corrente por 24 horas e núculas intactas como controle. As núculas (50 por tratamento) foram semeadas a $1 \mathrm{~cm}$ de profundidade, em vermiculita umedecida com água destilada à capacidade de campo, contida em bandejas de plástico. O teste de emergência foi conduzido sob luz branca fluorescente contínua, a $21,8 \mu \mathrm{mol} \mathrm{m}^{-2} \mathrm{~s}^{-1} \mathrm{e} 25^{\circ} \mathrm{C}$. O percentual de emergência apresentou distribuição normal aproximada para todos os tratamentos que, quando comparados pelo teste $t$ de “Student”, não diferiram significativamente (emergência entre 70\% e 76\%). Plântulas emergidas a partir de núculas escarificadas e posteriormente lavadas apresentaram menor tempo médio (16,3 dias) e conseqüentemente maior velocidade média de emergência $\left(0,0613 \mathrm{dia}^{-1}\right)$ em relação aos demais tratamentos. Os valores de incerteza distantes de zero ( $I \geq 3,28$ bits) e de sincronia próximos a zero $(Z \leq 0,095)$ indicam baixa sincronia de emergência das plântulas da espécie. Os resultados mostraram que, mesmo com um número reduzido de núculas, foi possível inferir sobre o processo de emergência das plântulas da espécie, sem violação dos princípios e pressuposições estatísticas.

Palavras-chave - cajuí, distribuição normal, medidas de emergência, teste t de "Student”

\section{Introdução}

Algumas espécies nativas apresentam problemas de compatibilidade nos cruzamentos, sazonalidade na produção de frutos e sementes e ainda podem sofrer o

\footnotetext{
1. Universidade Federal de Uberlândia, Instituto de Ciências Agrárias, Caixa Postal 593, 38400-902 Uberlândia, MG, Brasil.

2. Universidade Federal de Uberlândia, Instituto de Biologia, Caixa Postal 593, 38400-902 Uberlândia, MG, Brasil.

3. Autor para correspondência: dgsantana@umuarama.ufu.br
}

efeito de ações antrópicas, principalmente na fase reprodutiva. Essas limitações dificultam o estabelecimento de métodos para experimentos de germinação e emergência de plântulas, principalmente no que se refere ao número de sementes (ou unidades de dispersão) por amostra ou por tratamento. Para espécies cultivadas, os padrões para testes laboratoriais de germinação estão descritos nas Regras para Análise de Sementes (Brasil 1992) e são amplamente utilizados por tecnologistas de sementes. Nessas regras, o número mínimo de sementes por amostra é de 400, o que é muito alto para algumas espécies nativas. Acrescenta-se a 
isso, as limitações inerentes aos modelos experimentais, como os delineamentos inteiramente casualizados e de blocos casualizados, que exigem no mínimo 20 parcelas (produto entre número de tratamentos e número de repetições) e 10 graus de liberdade para o resíduo ou para o erro experimental, para garantirem a representatividade dos dados obtidos (Banzatto \& Kronka 1989, Pimentel-Gomes 1990). Quando o número de sementes não atende aos requisitos dessas técnicas experimentais de delineamento, algumas das opções são os testes t de "Student" e Mann-Whitney para comparações entre duas amostras ou dois tratamentos, que não são restringidos pelo tamanho da amostra. Amplamente descritos nos livros de estatística clássica (Vieira 1980, Sokal \& Rohlf 1997, Zar 1999), esses testes permitem comparações entre amostras ou tratamentos, o primeiro para variáveis com distribuição normal ou normal aproximada e o segundo para variáveis com outra distribuição (Snedecor \& Cochran 1989, Sokal \& Rohlf 1997).

Algumas espécies nativas como Anacardium humile A. St.-Hil., conhecido popularmente como cajuí, cajuzinho-do-cerrado ou cajuzinho-do-campo (Rede de Sementes do Cerrado 2005), apresentam baixa capacidade de produção de frutos e sementes. Segundo Almeida et al. (1998), essa baixa produção da espécie ocorre devido à alta proporção entre flores estaminadas e monóclinas (4:1). As flores monóclinas apresentam ovários súperos, uniloculares (Barroso et al. 1999), com um só óvulo lateral e, nas flores estaminadas, apenas um, em cada oito ou 10 estames, é fértil (Almeida et al. 1998). Associam-se a essas características, limitações na polinização, como a tendência dos grãos de pólen de se manterem aderentes à antera, mesmo depois da deiscência, e a incapacidade de algumas flores monóclinas em se transformarem em fruto, apesar do grande número de flores por inflorescência (Ferrão 1995).

A espécie ocorre com freqüência em campo sujo e cerrado sensu stricto, sendo as principais áreas de distribuição os estados de Rondônia, Bahia, Goiás, Distrito Federal, Mato Grosso, Mato Grosso do Sul, Minas Gerais, Paraná e São Paulo (López-Naranjo \& Pernía 1990, Almeida et al. 1998). As núculas dessa espécie possuem uma das características mais marcantes da família Anacardiaceae, que são canais ou cavidades de goma-resina, bastante desenvolvidos e geralmente associados ao floema, ocupando quase todo o mesocarpo (Machado \& Carmello-Guerreiro 2001). O fruto verdadeiro é uma núcula reniforme, com pericarpo duro e seco, de cor parda, alcançando o seu tamanho final antes mesmo do pedicelo se tornar espessado e modificado na forma de uma baga (Barroso et al. 1999). Segundo descrição das autoras, a semente é reniforme, de inserção basal, preenchendo toda a cavidade da núcula; o embrião é carnoso e curvo, não possuindo endosperma.

A planta é heliófila (Hoehne 1979) e melífera (Almeida et al. 1998), apresentando potencial medicinal e alimentar, podendo ser enquadrada no grupo das fruteiras tropicais. O falso fruto, um pouco mais ácido, quando comparado ao do cajueiro comum, e os frutos, são valorizados pelas populações locais como fonte alimentar (Corrêa 1984, Almeida et al. 1998). O óleo encontrado na castanha é corrosivo e volátil, contendo cardol e ácido anacárdico, considerados de uso medicinal, com ação antisséptica e cicatrizante (Barroso et al. 1999). A espécie é fonte alimentar de canídeos silvestres neotropicais, como o Lycalopex vetulus (raposa-do-campo), que atua como agente dispersor em áreas mais secas do cerrado e que, por apresentar elevada freqüência de sementes intactas nas suas fezes durante diferentes períodos do ano, pode ser considerado um dos recolonizadores de áreas abertas por atividade humana (Dalponte \& Lima 1999).

O hábito rasteiro torna a espécie mais susceptível a ações antrópicas e ao fogo, quando comparado ao cajueiro comum (Anacardium occidentale L.), concorrendo com outras espécies ao título de espécie ameaçada de extinção (Cemig 2001). Por outro lado, o xilopódio bem desenvolvido e com capacidade de armazenar água, garante resistência às secas prolongadas e queimadas, uma vez que após a perda da parte aérea, os brotos plagiotrópicos reativam-se, invertendo a direção de crescimento, sendo esta diferenciação rápida e ocorrendo antes que os órgãos regenerativos das gramíneas e herbáceas cubram a superfície do solo (López-Naranjo \& Pernía 1990).

As núculas de Anacardium humile não apresentam dormência e a longevidade é baixa, com a viabilidade diminuindo após um mês de armazenamento (Almeida et al. 1998). De maneira geral, os diásporos do gênero Anacardium possuem baixa capacidade de germinação, quando semeados sem qualquer pré-tratamento (Lorenzi 1998), embora existam registros de percentagem de germinação superior a $60 \%$ para núculas de $A$. humile, em um período de 20 dias (Melo et al. 1998).

Diante do exposto, o presente trabalho teve por objetivo introduzir a teoria estatística de amostras pequenas ao estudo da emergência de plântulas de Anacardium humile. 


\section{Material e métodos}

Coleta e beneficiamento - Pseudofrutos com as núculas de Anacardium humile A. St.-Hil. foram aleatoriamente coletados a partir de aproximadamente 50 indivíduos, na primeira semana de novembro de 2003, na Reserva Vegetal do Clube Caça e Pesca Itororó de Uberlândia, MG (1855’23” S e 48¹7’19” W), em área de cerrado sensu lato. O clima da região de Uberlândia é do tipo Aw, segundo o sistema de classificação de Köppen (1948), com verão chuvoso de outubro a março e inverno seco de abril a setembro.

Os frutos foram destacados manualmente dos pseudofrutos, previamente selecionados para a retirada dos malformados, predados e chochos e, rapidamente lavados, antes da instalação do experimento no Laboratório de Sementes Florestais (Lasef) do Instituto de Ciências Agrárias da Universidade Federal de Uberlândia.

Teste de emergência de plântulas - As 150 núculas coletadas foram equalizadas por tamanho e distribuídas em três tratamentos, cada um deles com 50 unidades. Os tratamentos constaram da escarificação por abrasão do pericarpo na região junto ao pedicelo; escarificação como descrito anteriormente e posterior lavagem em água corrente por 24 horas; e núculas intactas como controle. A semeadura foi feita a $1 \mathrm{~cm}$ de profundidade em bandejas de $30 \mathrm{~cm}$ de largura, $44 \mathrm{~cm}$ de comprimento e 8,5 cm de profundidade, contendo vermiculita expandida como substrato (volume de expansão de 0,1 $\mathrm{m}^{3}$ ). $\mathrm{O}$ substrato foi umedecido com água destilada à capacidade de campo e as bandejas foram dispostas aleatoriamente em câmara de germinação (Seedburo Equipment Company modelo MPG - 2000), sob luz branca fluorescente contínua (21,8 \pm $5,7 \mu \mathrm{mol} \mathrm{m}{ }^{-2} \mathrm{~s}^{-1}$ ), à temperatura de $25 \pm 2{ }^{\circ} \mathrm{C}$. As contagens foram realizadas diariamente e o critério de emergência adotado foi a exposição de qualquer parte da plântula na superfície do substrato. Ao final do experimento, as núculas que não germinaram foram submetidas ao teste do tetrazólio a $0,5 \%$.

Medidas analisadas - Calculou-se o percentual de emergência; tempo médio de emergência adaptado de Labouriau (1983); velocidade média de emergência adaptada de Labouriau (1970); velocidade de emergência (Maguire 1962); índice de Timson (Timson 1965), adaptado e índice de Timson modificado por Santana \& Ranal (2004); índice de velocidade de emergência (Shmueli \& Goldberg 1971) e índice de velocidade de emergência modificado por Santana \& Ranal (2004). A uniformidade e a heterogeneidade do processo de emergência foram calculadas pelo coeficiente de uniformidade de emergência adaptado de Heydecker (1973), pelo desvio padrão do tempo de emergência adaptado de Labouriau (1983) e pelo coeficiente de variação do tempo, $C V_{t}=\frac{s_{t}}{\bar{t}}$, adaptado de Pimentel-Gomes (1990), onde $s_{t}$ é o desvio padrão do tempo de emergência e $\bar{t}$ é o tempo médio de emergência. Medidas de sincronia foram calculadas pela incerteza (Labouriau \& Valadares 1976) e pelo índice de sincronização, $Z=\frac{c}{a}$, adaptado de Primack (1980), onde $c$ é o somatório das combinações diárias de sincronia e $a$ é a sincronia perfeita para o número de plântulas emergidas. Distribuições das freqüências relativas de emergência em relação ao tempo adaptadas de Labouriau \& Pacheco (1978) foram construídas para cada tratamento como um indicativo da distribuição teórica da emergência (normal ou outra) e da homogeneidade do processo.

Procedimento estatístico - A proporção de plântulas emergidas é uma variável discreta, uma vez que apenas dois resultados são possíveis (emerge ou não emerge) e para $n$ núculas, esta variável apresenta distribuição binomial. Metodologias estatísticas para comparações entre dois tratamentos foram desenvolvidas com o intuito de aplicar as técnicas de variáveis contínuas com distribuição normal aproximada, como a distribuição t de "Student", às variáveis discretas. Na literatura, esta metodologia é conhecida como aproximação da distribuição binomial pela normal e, para dados de percentagem ou proporção, esta aproximação ocorre quando $n_{1} \hat{p}_{1}>5, n_{1} \hat{q}_{1}>5, n_{2} \hat{p}_{2}>5$ e $n_{2} \hat{q}_{2}>5$, sendo $\hat{p}_{1}=\frac{X_{1}}{n_{1}}$; $\hat{q}_{1}=1-\hat{p_{1}} ; \hat{p_{2}}=\frac{X_{2}}{n_{2}}$ e $\hat{q_{2}}=1-\hat{p_{2}}$, onde $\hat{p_{1}}$ e $\hat{p_{2}}$ são as estimativas das proporções de plântulas emergidas no primeiro e segundo tratamentos, respectivamente; $X_{1}$ e $X_{2}$ o número de plântulas emergidas no primeiro e segundo tratamentos, respectivamente; $n_{1}$ e $n_{2}$, o número de núculas do primeiro e segundo tratamentos, respectivamente. Como estas condições de aproximação foram atendidas para o percentual de emergência das plântulas de Anacardium humile, a comparação entre pares de tratamentos foi feita de acordo com a seguinte expressão:

$$
t=\frac{\hat{p}_{1}-\hat{p}_{2}}{\sqrt{\frac{\hat{p}_{1} \hat{q}_{1}}{n_{1}}+\frac{\hat{p}_{2} \hat{q}_{2}}{n_{2}}}}
$$

A variabilidade de algumas variáveis, quando desconhecida, pode ser estimada pelos dados amostrais (Neter et al. 1985, Sokal \& Rohlf 1997). Desta forma, comparações entre tempos médios de emergência foram feitas pelo teste t de "Student", com base na variância do tempo, $s_{t}^{2}$ (Labouriau 1983), segundo a expressão

$$
t=\bar{t}_{1}-\bar{t}_{2} / \sqrt{\frac{s_{t_{1}}^{2}}{\mathrm{n}_{1}}+\frac{s_{t_{2}}^{2}}{\mathrm{n}_{2}}}
$$

onde $\bar{t}_{1}$ e $\bar{t}_{2}$ são os tempos médios de emergência do primeiro e segundo tratamentos, respectivamente; $s_{t_{1}}^{2}$ e $s_{t_{2}}^{2}$ as variâncias do tempo do primeiro e segundo tratamentos, respectivamente; e $n_{1}$ e $n_{2}$, o número de plântulas emergidas no primeiro e segundo tratamentos, respectivamente.

As velocidades médias de emergência foram avaliadas pelo teste t de "Student", com base na variância apresentada por Labouriau (1983) para esta variável. Como a velocidade média não é uma medida linear $(\bar{v}=1 / \bar{t})$, a estimativa da variância é aproximada e foi obtida pelo desenvolvimento de uma expansão em série de Taylor, sendo expressa por , $s_{v}^{2}=(\bar{v})_{2}^{4} s_{t}^{2}$, onde $\bar{v}$ é a velocidade média de emergência e 
$\mathrm{s}_{t}^{2}$ variância do tempo de emergência (Labouriau 1983). Com a medida de variabilidade, as comparações entre os pares de tratamentos para a velocidade média de emergência foram executadas com base na expressão

$$
t=\bar{v}_{1}-\bar{v}_{2} / \sqrt{\frac{s_{v_{1}}^{2}}{\mathrm{n}_{1}}+\frac{s_{v_{2}}^{2}}{\mathrm{n}_{2}}},
$$

onde $\bar{v}_{1}$ e $\bar{v}_{2}$ são as velocidades médias de emergência do primeiro e segundo tratamentos, respectivamente; $s_{v_{1}}^{2}$ e $s_{v_{2}}^{2}$ as variâncias da velocidade do primeiro e segundo tratamentos, respectivamente; e $\mathrm{n}_{1}$ e $\mathrm{n}_{2}$, o número de plântulas emergidas no primeiro e segundo tratamentos, respectivamente. Comparações entre tratamentos quanto à incerteza $(I)$ foram feitas segundo a metodologia apresentada por Zar (1999) e Hutcheson (1970). Como as medidas do coeficiente de uniformidade de emergência (CUG), coeficiente de variação do tempo $\left(C V_{\mathrm{t}}\right)$, velocidade de emergência $(V E)$, índice de Timson $(T)$, índice de Timson modificado $\left(T_{\text {mod }}\right)$, índice de velocidade de emergência $(E R I)$, índice de velocidade de emergência modificado $\left(E R I_{\bmod }\right)$ e índice de sincronização $(Z)$ não apresentam variabilidades associadas, não podendo ser estimadas pelos dados amostrais, a discussão dos resultados para essas variáveis foi apenas descritiva. Em todas as análises foi utilizado $\alpha=0,05$ como valor de significância.

\section{Resultados}

A emergência das plântulas iniciou-se 11 dias após a semeadura, estabilizando-se 17 dias após a primeira emergência, perfazendo um total de 38 dias entre a semeadura e o tempo final. Os percentuais de emergência apresentaram distribuição normal aproximada para todos os tratamentos $(n \hat{p}>5 \mathrm{e}$ $n q$ > 5) e, quando comparados pelo teste t de "Student", não diferiram significativamente $(P>0,05)$, variando entre $70 \%$ e $76 \%$ (tabela 1 ). As freqüências relativas de emergência mostram que, mesmo com um número reduzido de núculas, a distribuição observada se aproxima da distribuição gaussiana ou normal (figura 1).

Plântulas emergidas a partir de núculas escarificadas e posteriormente lavadas por $24 \mathrm{~h}$ em água corrente apresentaram menor tempo médio de emergência ( $\bar{t}=16,3$ dias) e, como conseqüência, a velocidade média de emergência foi maior neste tratamento $\left(\bar{v}=0,0613 \mathrm{dia}^{-1}\right)$, quando comparada à velocidade de emergência de núculas intactas.

A dispersão da emergência ao longo do tempo foi medida pelo desvio padrão do tempo de emergência $\left(s_{t}\right)$,

Tabela 1. Medidas de emergência de plântulas de Anacardium humile A. St.-Hil. provenientes de núculas coletadas na Reserva Vegetal do Clube Caça e Pesca Itororó, Uberlândia, MG, novembro de 2003. *Valores seguidos por letras distintas (linha) diferem entre si pelo teste t de "Student" $(P<0,05)$.

Table 1. Emergence measurements of the Anacardium humile A. St.-Hil. seedlings resulting from cashew nuts collected at the Vegetal Reserve of the “Caça e Pesca Itororó” Club, Uberlândia, MG, November 2003. *Values followed by the same letter in each line are not significantly different based on the Student t test $(P<0.05)$.

\begin{tabular}{|c|c|c|c|}
\hline \multirow{2}{*}{ Medida (unidade) } & \multicolumn{3}{|c|}{ Tratamento* } \\
\hline & \multirow{2}{*}{$\begin{array}{c}\begin{array}{c}\text { Núcula } \\
\text { intacta }\end{array} \\
74,0 \pm 6,2 \text { a }\end{array}$} & \multirow{2}{*}{$\begin{array}{c}\begin{array}{c}\text { Núcula } \\
\text { escarificada }\end{array} \\
76,0 \pm 6,0 \text { a }\end{array}$} & \multirow{2}{*}{$\begin{array}{c}\begin{array}{c}\text { Escarificada } \\
\text { e lavada }\end{array} \\
70,0 \pm 6,5 \text { a }\end{array}$} \\
\hline G: emergência (\%) & & & \\
\hline $\bar{t}:$ tempo médio de emergência (dia) & $19,27 \mathrm{~b}$ & $17,58 \mathrm{ab}$ & $16,31 \mathrm{a}$ \\
\hline$s_{t}:$ desvio padrão do tempo de emergência (dia) & 6,615 & 4,500 & 5,905 \\
\hline CUG: coeficiente de uniformidade de emergência $\left(\right.$ dia $\left.^{-2}\right)$ & 0,0235 & 0,0507 & 0,0295 \\
\hline$C V_{\mathrm{t}}:$ coeficiente de variação do tempo (\%) & 34,33 & 25,60 & 36,20 \\
\hline $\bar{V}:$ velocidade média de emergência $\left(\mathrm{dia}^{-1}\right)$ & $0,0519 b$ & $0,0569 \mathrm{ab}$ & 0,0613 a \\
\hline$V E$ : velocidade de emergência (plântula dia $^{-1}$ ) & 2,090 & 2,262 & 2,318 \\
\hline T: índice proposto por Timson (\% dia) & 1460,0 & 1628,0 & 1518,0 \\
\hline$T_{\text {mod }}:$ índice proposto por Timson e modificado (dia) & 19,733 & 21,421 & 21,686 \\
\hline ERI: índice de velocidade de emergência (plântula dia) & 693,0 & 776,0 & 724,0 \\
\hline$E R I_{\bmod }$ índice de velocidade de emergência modificado (dia) & 18,730 & 20,421 & 20,686 \\
\hline I: $\quad$ incerteza (bit) & 3,715 a & $3,281 \mathrm{a}$ & $3,297 \mathrm{a}$ \\
\hline Z: índice de sincronização & 0,0601 & 0,0953 & 0,0908 \\
\hline
\end{tabular}



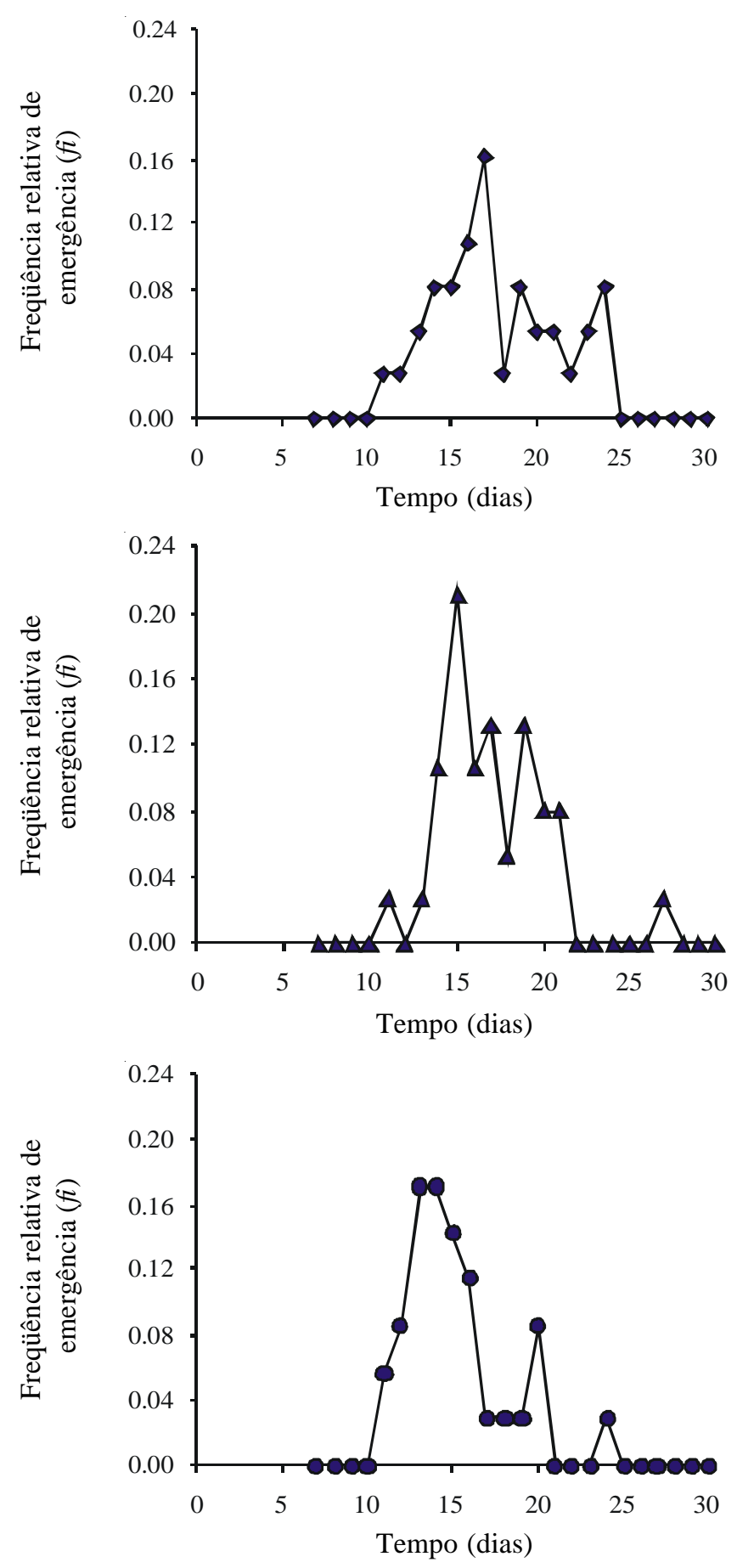

Figura 1. Distribuição da frequiência relativa de emergência $\left(f_{i}\right)$ de plântulas de Anacardium humile A. St.-Hil. em relação ao tempo de emergência de núculas intactas, escarificadas e escarificadas seguidas de lavagem em água corrente por 24 horas. $\boldsymbol{\nabla}$ = intactas, $\boldsymbol{\Delta}=$ escarificadas, $\boldsymbol{\bullet}=$ escarificadas $\mathrm{e}$ lavadas.

Figure 1. Relative frequency distribution of the emergence $\left(f_{i}\right)$ of Anacardium humile A. St.-Hil. seedlings in relation to time of emergence of intact cashew nuts, scarified and scarified followed by washing in water during 24 hours. $\bullet$ = intact, $\boldsymbol{\Delta}=$ scarified, $\boldsymbol{\bullet}=$ scarified and washed. pelo coeficiente de uniformidade de emergência ( $C U G)$ e pelo coeficiente de variação do tempo $\left(C V_{\mathrm{t}}\right)$. Essas três medidas de variabilidade mostraram que a emergência de plântulas foi mais homogênea quando as núculas foram escarificadas, uma vez que foram registrados baixos valores para o desvio padrão e coeficiente de variação do tempo e alto valor para o coeficiente de uniformidade (tabela 1). A relação entre o tempo de emergência e a freqüência relativa de emergência (figura 1) indicou que núculas intactas são mais desuniformes, com maior espalhamento da emergência em torno do tempo médio.

Analisando-se descritivamente a velocidade de emergência $(V E)$, o índice de Timson modificado $\left(T_{\text {mod }}\right)$ e o índice de velocidade de emergência modificado $\left(E R I_{\text {mod }}\right)$, pode-se verificar que essas medidas apontaram a mesma sequiência detectada pela velocidade média de emergência $(\bar{v})$, para os tratamentos com maior e menor velocidade. $O$ índice de Timson $(T)$ e o índice de velocidade de emergência $(E R I)$ apresentaram seqüência diferente de $\bar{v}$, ainda que tenham, da mesma forma, indicado velocidade menor para núculas intactas (tabela 1).

Os valores de incerteza distantes de zero $(I \geq 3,28)$ e de $Z$ próximos a zero $(Z \leq 0,095)$ indicam baixa sincronia de emergência das plântulas da espécie (tabela 1).

\section{Discussão}

Os percentuais de emergência das plântulas de Anacardium humile A. St.-Hil. foram próximos aos encontrados por Melo et al. (1998) para a germinação de núculas da espécie. Mesmo que a literatura relate a presença de inibidores nos diásporos do gênero Anacardium (Lorenzi 1998), o percentual de emergência das plântulas de Anacardium humile foi alto sem qualquer pré-tratamento. As núculas não germinadas apresentaram danos causados por insetos em partes vitais do embrião, diagnosticado pelo teste do tetrazólio, prejudicando sua viabilidade. Para núculas de Anacardium occidentale, $100 \%$ de germinação foram alcançados (Rao et al. 1957, Subbaiah 1982), mas, de acordo com a procedência dos diásporos, este percentual pode ser menor (Rocchetti \& Panerai 1968).

Mesmo com o pequeno número de núculas disponíveis em cada tratamento, o percentual de emergência apresentou distribuição normal aproximada, característica das medidas provenientes de amostras grandes, justificando a aplicação da estatística t de "Student". Este resultado amplia as possibilidades de 
análise estatística para testes de germinação e emergência de plântulas de espécies com baixa produção de sementes ou produção sazonal. Apesar do teste $t$ de "Student" para comparações entre proporções estar amplamente descrito na literatura (Costa Neto 1977, Vieira 1980, Snedecor \& Cochran 1989, Sokal \& Rohlf 1997, Zar 1999), ainda há resistência quanto ao uso de amostras pequenas em experimentos de germinação e emergência. Os resultados mostraram que, mesmo com um número reduzido de núculas, é possível fazer inferências sobre o processo de emergência das plântulas da espécie, sem violação dos princípios e pressuposições estatísticas. Essa técnica estatística também abre enorme possibilidade para estudos de biologia floral, no que se refere ao sucesso reprodutivo, em que o número de sementes obtido por meio de cruzamentos específicos é muito baixo, limitando a comunicação das informações obtidas sobre viabilidade e/ou vigor da geração $F_{1}$ dos cruzamentos.

Como o desvio padrão do tempo e o coeficiente de uniformidade de emergência são medidas matematicamente influenciadas pelo tempo médio (Santana \& Ranal 2004), apenas quando os tempos médios de emergência são iguais, a comparação entre amostras ou entre tratamentos pode indicar qual amostra ou tratamento apresenta emergência mais homogênea. Por outro lado, como o coeficiente de variação do tempo é uma medida de variabilidade relativa do tempo médio, este proporciona maior confiabilidade, ainda que descritivamente, da uniformidade de emergência. Isto significa que, independentemente do tempo médio de emergência, núculas escarificadas foram as mais homogêneas, seguidas das núculas intactas e por fim pelas escarificadas e lavadas. Uma relação entre os coeficientes de variação do tempo de emergência das plântulas de Anacardium humile e valores de referência do coeficiente de variação para experimentos planejados em delineamentos experimentais (Pimentel-Gomes 1990) indica que a emergência das plântulas dessa espécie apresenta alta variabilidade, por terem sido registrados valores de $C V_{\mathrm{t}}$ acima de $20 \%$.

Apesar da dormência ser a característica do processo de germinação mais relatada para justificar a adaptação das espécies às condições adversas do ambiente, principalmente das espécies nativas (Labouriau 1983, Bewley \& Black 1994, Baskin \& Baskin 1998), outras características do processo também podem estar associadas a esta adaptação. Espécies com sementes não dormentes aumentam as chances de estabelecimento no ambiente quando, após a dispersão, permanecem por um longo período capazes de gerar plântulas normais. As plântulas de Anacardium humile gastaram 38 dias para estabilizarem a emergência, sendo, portanto, capazes de garantirem que o processo ocorra em condições mais adequadas para seu estabelecimento. As núculas, ainda que não dormentes, espalharam no tempo a emergência (altos valores de $C V_{\mathrm{t}}$ ), ou seja, apresentaram baixa freqüência de plântulas emergidas num mesmo intervalo de tempo (baixa sincronia), característica que também favorece a adaptação da espécie.

A diminuição do tempo médio e o conseqüente aumento na velocidade média com a escarificação e lavagem das núculas, em relação às intactas, indicaram que a pré-embebição acelera a emergência das plântulas. Núculas de Anacardium occidentale L. pré-embebidas em água também apresentam germinação mais sincronizada, quando comparada às núculas intactas (Subbaiah 1982).

Ainda com relação ao método estatístico proposto, é preciso destacar que, para variáveis expressas em percentagem (como é o caso da percentagem de emergência) ou expressas por valores médios obtidos de uma única amostra de tamanho $n$ (como é o caso de $\bar{t}$ e $\bar{v}$ ), o teste $\mathrm{t}$ de "Student" só é aplicável quando a variabilidade pode ser estimada, mesmo que por aproximação, pelos dados amostrais. Para variáveis com variabilidades desconhecidas, e que não podem ser estimadas pela amostra (como é o caso do $V E, T, T_{\text {mod }}$, $E R I$ e $E R I_{\text {mod }}$ ), a interpretação dos resultados limita-se à análise descritiva, sendo esta uma desvantagem do método de amostragem e a sua limitação.

Agradecimentos - À Marcela Bueno de Mattos pela ajuda nas atividades de laboratório e à Diretoria do Clube Caça e Pesca Itororó pela permissão para a coleta dos frutos.

\section{Referências bibliográficas}

ALMEIDA, S.P., PROENÇA, C.E.B., SANO, S.M. \& RIBEIRO, J.F. 1998. Cerrado: espécies vegetais úteis. Embrapa-Cpac, Planaltina.

BANZATTO, D.A. \& KRONKA, S.N. 1989. Experimentação agrícola. Funep, Jaboticabal.

BARROSO, G.M., MORIM, M.P., PEIXOTO, A.L. \& ICHASO, C.L.F. 1999. Frutos e sementes: morfologia aplicada à sistemática de dicotiledôneas. Editora Ufv, Viçosa.

BASKIN, C.C. \& BASKIN, J.M. 1998. Seeds: ecology, biogeography, and evolution of dormancy and germination. Academic Press, San Diego.

BEWLEY, J.D. \& BLACK, M. 1994. Seeds: physiology of development and germination. Plenum Press, New York. 
BRASIL. 1992. Ministério da Agricultura e Reforma Agrária. Regras para análise de sementes. Snda/Dndv/Clav, Brasília.

CEMIG. 2001. Companhia Energética de Minas Gerais. Assessoria de coordenação ambiental. Guia ilustrativo de plantas do cerrado de Minas Gerais. Nobel, São Paulo.

CORRÊA, M.P. 1984. Dicionário das plantas úteis do Brasil e das exóticas cultivadas. Imprensa Nacional, Ministério da Agricultura, Instituto Brasileiro de Desenvolvimento Florestal, Rio de Janeiro.

COSTA NETO, P.L.O. 1977. Estatística. Edgard Blücher, São Paulo.

DALPONTE, J.C. \& LIMA, E.S. 1999. Disponibilidade de frutos e a dieta de Lycalopex vetulus (CarnivoraCanidae) em um cerrado de Mato Grosso, Brasil. Revista Brasileira de Botânica 22:325-332.

FERRÃO, J.E.M. 1995. O cajueiro (Anacardium occidentale L.). Instituto de Investigação Cientifica Tropical, Lisboa.

HEYDECKER, W. 1973. Glossary of terms. In Seed Ecology (W. Heydecker, ed.). Butterworths, London, p.553-557.

HOEHNE, F.C. 1979. Enumeração das principais espécies de fruteiras indígenas, pela ordem alfabética de suas famílias. In Frutas indígenas (F.C. Hoehne, ed.). Instituto de Botânica, São Paulo, p.13-79.

HUTCHESON, K. 1970. A test for comparing diversities based on the Shannon formula. Journal of Theoretical Biology 29:151-154.

KÖPPEN, W. 1948. Climatología: con un estudio de los climas de la tierra. Fondo de Cultura Econômica, Mexico.

LABOURIAU, L.G. 1970. On the physiology of seed germination in Vicia graminea Sm.-I. Anais da Academia Brasileira de Ciências 42:235-262.

LABOURIAU, L.G. 1983. A germinação das sementes. Secretaria Geral da OEA, Washington.

LABOURIAU, L.G. \& PACHECO, A.A. 1978. On the frequency isothermal germination in seeds of Dolichos biflorus L. Plant \& Cell Physiology 19:507-612.

LABOURIAU, L.G. \& VALADARES, M.E.B. 1976. On the germination of seeds of Calotropis procera (Ait.) Ait. $\mathrm{f}$. Anais da Academia Brasileira de Ciências 48:263-284.

LÓPEZ-NARANJO, H. \& PERNÍA, N.E. 1990. Anatomia y ecologia de los organos subterraneos de Anacardium humile A. St.-Hil. (Anacardiaceae). Revista Forestal Venezolana 24:55-76.

LORENZI, H. 1998. Árvores brasileiras: manual de identificação e cultivo de plantas arbóreas nativas do Brasil. Editora Plantarum, Nova Odessa.

MACHADO, S.R. \& CARMELLO-GUERREIRO, S.M. 2001. Estrutura e desenvolvimento de canais secretores em frutos de Schinus terebinthifolius Raddi (Anacardiaceae). Acta Botanica Brasilica 15:189-195.
MAGUIRE, J.D. 1962. Speed germination-aid in selection and evaluation for seedling emergence and vigor. Crop Science 2:176-177.

MELO, J.T., SILVA, J.A., TORRES, R.A.A., SILVEIRA, C.E.S. \& CALDAS, L.S. 1998. Coleta, propagação e desenvolvimento inicial de espécies do cerrado. In Cerrado: ambiente e flora (S.M. Sano \& S.P. Almeida, eds.). Embrapa-Cpac, Planaltina, p.195-243.

NETER, J., WASSERMAN, W. \& KUTNER, M.H. 1985. Applied linear statistical models. Richard D. Irwin, Illinois.

PIMENTEL-GOMES, F. 1990. Curso de estatística experimental. Degaspari, Piracicaba.

PRIMACK, R.B. 1980. Variation in the phenology of natural populations of montane shrubs in New Zealand. Journal of Ecology 68:849-862.

RAO, V.N.M., RAO, I.K.S. \& HASSAN, M.V. 1957. Studies on certain aspects of germination of seeds in cashew (Anacardium occidentale Linn.). The Indian Journal of Agricultural Science 37:25-34.

REDE DE SEMENTES DO CERRADO. 2005. Rede de sementes do Cerrado: Anacardium humile A. St.-Hil. http://www.sementesdocerrado.bio.br (acesso em 04/05/2005).

ROCCHETTI, G. \& PANERAI, L. 1968. L'effetto delle temperature sulla germinazione delle castagne di anacardio. Revista di Agricoltura Subtropicale e Tropicale 62:228-235.

SANTANA, D.G. \& RANAL, M.A. 2004. Análise da germinação: um enfoque estatístico. Editora Universidade de Brasília, Brasília.

SHMUELI, M. \& GOLDBERG, D. 1971. Emergence, early growth, and salinity of five vegetable crops germinated by sprinkle and trickle irrigation in an arid zone. HortScience 6:563-565.

SNEDECOR, G.W. \& COCHRAN, W.G. 1989. Statistical methods. Iowa State University Press, Ames.

SOKAL, R.R. \& ROHLF, F.J. 1997. Biometry. W.H. Freeman and Company, New York.

SUBBAIAH, C.C. 1982. Effect of pre-soaking in organic solvents on seed germination and seedling growth of cashew. Scientia Horticulturae 18:137-142.

TIMSON, J. 1965. New method of recording germination data. Nature 207:216-217.

VIEIRA, S. 1980. Introdução à bioestatística. Campus, Piracicaba.

ZAR, J.H. 1999. Biostatistical analysis. Prentice-Hall, New Jersey. 